\title{
A sealed ceramic GEM-based neutron detector
}

\author{
Jianjin Zhou ${ }^{\text {a,b,c }}$, Jianrong Zhou ${ }^{\text {b,c, }}{ }^{* *}$, Xiaojuan Zhou ${ }^{\text {b,c }}$, Lin Zhu ${ }^{\text {b,c }}$, Yangdong Wei ${ }^{\mathrm{d}}$, Hong Xu ${ }^{\text {b,c }}$, \\ Huiyin $\mathrm{Wu}{ }^{\text {e }}$, Kang Wei ${ }^{\text {a }}$, Jianqing Yang ${ }^{\text {b,c }}$, Guian Yang ${ }^{\text {b,c }}$, Yuguang Xie ${ }^{\mathrm{c}}$, Yi Zhang ${ }^{\mathrm{a}}$, Xiaohu

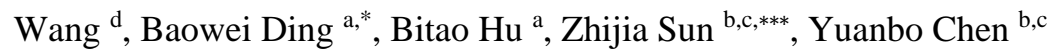 \\ a School of Nuclear Science and Technology, Lanzhou University, Lanzhou, 730000, China \\ b Spallation Neutron Source Science Center, Dongguan 523803, Guangdong, China \\ c State Key Laboratory of Particle Detection and Electronics, Institute of High Energy Physics, Chinese Academy \\ of Sciences, Beijing 100049, China \\ d School of National Defense Science and Technology, Southwest University of Science and Technology, \\ Mianyang 621000, China \\ e Chongqing Jian'an Instrument Co., Ltd., Chongqing 400060, China
}

\begin{abstract}
The GEM-based neutron detector has flourished in the past decade. However almost all the GEM-based neutron detectors work in the flow-gas mode, and the long-term performances of the detectors may be unstable due to the dynamic changes of atmospheric pressure and ambient temperature. In this paper, a sealed ceramic GEM-based neutron detector was developed at China Spallation Neutron Source (CSNS) and its sensitive area was $100 \mathrm{~mm} \times 100 \mathrm{~mm}$. The characterizations of the detector were presented and discussed, which included the plateau curve, the neutron beam profile, the neutron wavelength spectrum, the spatial resolution (FWHM: $2.77 \pm 0.01$ (stat) $\mathrm{mm}$ ), the two-dimensional (2D) imaging ability, the neutron detection efficiency and the counting rate instability (Relative Standard Deviation (RSD): $0.7 \%$ ). The results show that the detector has good performances in sealed mode, and it can be used for the measurement of the direct neutron beam at CSNS.
\end{abstract}

Keywords: Sealed detector, Neutron detector, Ceramic GEM, Spallation neutron source

*Corresponding author: Baowei Ding, dingbw@lzu.edu.cn, Lanzhou, China

**Corresponding author: Jianrong Zhou, zhoujr@ihep.ac.cn, Dongguan, China

***Corresponding author: Zhijia Sun, sunzj@ihep.ac.cn, Beijing, China

\section{Introduction}

CSNS [1] is a high-flux pulsed spallation neutron source which is similar to SNS in the USA [2], J-PARC in Japan [3], ISIS in the UK [4] and ESS in Europe [5] and it has been in public operation at $100 \mathrm{~kW}$ power and $25 \mathrm{~Hz}$. At present, there are three neutron scattering instruments which have been built and seventeen which are being planned at CSNS. Efficient neutron detectors are an important component for every neutron scattering instrument. The dominant detectors are currently ${ }^{3} \mathrm{He}$-based gas detectors, such as Linear Position-Sensitive Detectors (LPSD) and MultiWire Proportional Chambers (MWPC). The counting rate of these detectors is a few tens $\mathrm{kHz}$, which is limited by the space charge accumulation near the wire [6]. In addition, the price of ${ }^{3} \mathrm{He}$ has been 
rising because of the ${ }^{3} \mathrm{He}$ crisis in 2008 [7]. It is necessary to develop alternative neutron conversion materials and detector structures.

Boron-10 has a larger absorption cross section at energies of thermal $\left(\sim 4 \times 10^{3} \mathrm{~b}\right)$ and epithermal $\left(\sim 10^{2}\right.$ b) neutrons than that of the other alternative neutron conversion materials such as Li. The boron converter layer made by evaporation or sputtering techniques is easily obtained, stable in physical and chemical properties, economic and environmental. The Gas Electron Multiplier (GEM) [8] detector, one of the micro-pattern gas detectors, offers excellent rate capability, good spatial resolution and time properties, radiation hardness and the possibility for large areas fabrication, and it is widely applied to high-energy physics. In the last decades, there was a booming development in GEM-based neutron detector by using boron conversion material [9]-[16]. The CASCADE neutron detector was firstly developed by Dr. Klein and Dr. Schmidt of Heidelberg University in Germany [9]. In addition to the standard GEM with $60 \mu \mathrm{m}$ thickness, the GEM also derived many types, such as FR4 GEM with 400-800 $\mu \mathrm{m}$ thickness in Israel [17], ceramic GEM with $200 \mu \mathrm{m}$ thickness in China [18], Liquid Crystal Polymer (LCP) GEM [19] and Low Temperature Co-fired Ceramic (LTCC) GEM [20] with $100 \mu \mathrm{m}$ thickness in Japan. At present, almost all the GEM-based neutron detectors work in flow-gas mode. Changes in atmospheric pressure and ambient temperature display daily and seasonal variations, the performances of detector may be unstable for long term. Meanwhile, the detector is not portable and not economical because of the gas system and gas consumption. In this paper, a sealed ceramic GEM-based neutron detector with a sensitive area of $100 \mathrm{~mm} \times 100 \mathrm{~mm}$ was developed and the neutron irradiation test of this detector was carried out at CSNS.

\section{Detector setup}

The sealed ceramic GEM-based neutron detector had the similar structure with the flow-gas GEM-based detector. A schematic of detector was shown in Fig. 1. The detector was composed of boron-coated cathode, ceramic GEM and 2D signal readout board. The ions produced by the reaction of ${ }^{10} \mathrm{~B}(\mathrm{n}, \alpha)^{7} \mathrm{Li}$ generate primary electrons in drift region, the electrons are avalanched in GEM hole due to strong electric field, and the signals are induced in 2D readout board as a result of the movement of electrons in induction region. To ensure the long-term stable performances of the detector, on one hand, the gas volume of the detector must be isolated from the outside without gas exchange; on the other hand, the outgassing rate of the materials in the gas volume must be extremely low. The detailed specifications of some key components are elaborated.

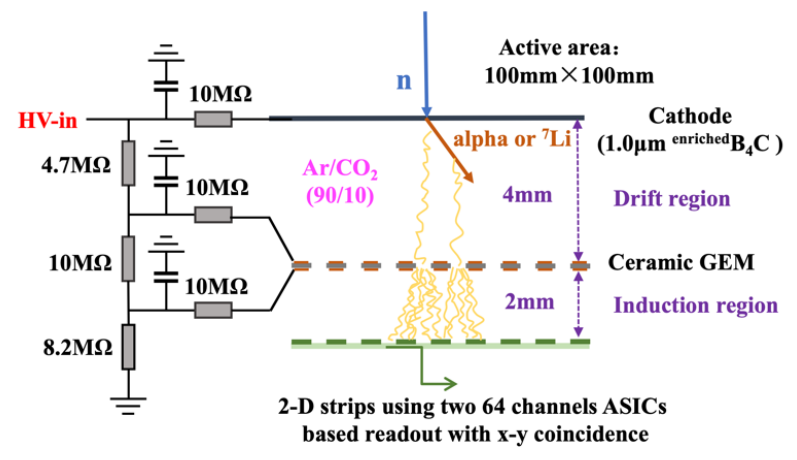

Fig. 1 Schematic of the detector. 
The detector was a sandwich-like circular structure in the middle of which there was a stainlesssteel flange used for welding high voltage feedthroughs, signal feedthroughs and gas tube. One side of the flange was the gas volume, and the other side was the electronics chamber. The gas volume was shown in Fig. 2(a), The high gas purity was maintained with the usage of ceramic materials for GEM, frames and screws which was a low-outgassing material. The cathode was a $300 \mu \mathrm{m}$ thick aluminum ( $\mathrm{Al})$ plate which was coated with a $1.0 \mu \mathrm{m}$ thick $\mathrm{B}_{4} \mathrm{C}\left({ }^{10} \mathrm{~B}\right.$ abundance: $\left.96 \%\right)$. The ceramic GEM with $100 \mathrm{~mm} \times 100 \mathrm{~mm}$ sensitive area was a double-copper coated ceramic foil of $200 \mu \mathrm{m}$ thickness, the $200 \mu \mathrm{m}$ diameter holes with a pitch of $600 \mu \mathrm{m}$ were drilled and the $80 \mu \mathrm{m}$ hole rims were etched by the printed circuit board technology. The ceramic GEM had higher transmittance and less self-scattering than FR4 GEM especially for cold neutrons, its performances were studied in Ref [18]. And the ceramic GEM had advantages including low outgassing, heatable and stable performances. The material of flexible readout board was Kapton and the thickness was about 0.6 $\mathrm{mm}$. To obtain the neutron position, there were 128 cross-strip channels with a strip pitch of 1.56 $\mathrm{mm}$ on readout board, where 64 channels were assigned to $\mathrm{x}$ direction and 64 to y direction. The gas tube which was controlled by a needle valve was used for a vacuum environment and a pure gas filling. All components of the gas volume were sealed by an Al gasket, which ensured that the gas volume was isolated from the air. The electronics chamber was shown in Fig. 2(b). By the signal transfer board and the signal feedthroughs, the signals with 128 channels were connected to two CPix ASIC chips (64 channels) [21] developed by Heidelberg ASIC lab. There was a very fast readout electronics system, based on analogue front-end readout chips in combination with modern Field-Programmable-Gate-Array (FPGA) technology. It was realized through the coincidence of correlation of signals in the time and space domain, and the detailed algorithm of neutron event reconstruction was described in Ref [16]. The high voltage board provided a suitable working voltage for the cathode and the ceramic GEM through a resistive chain.
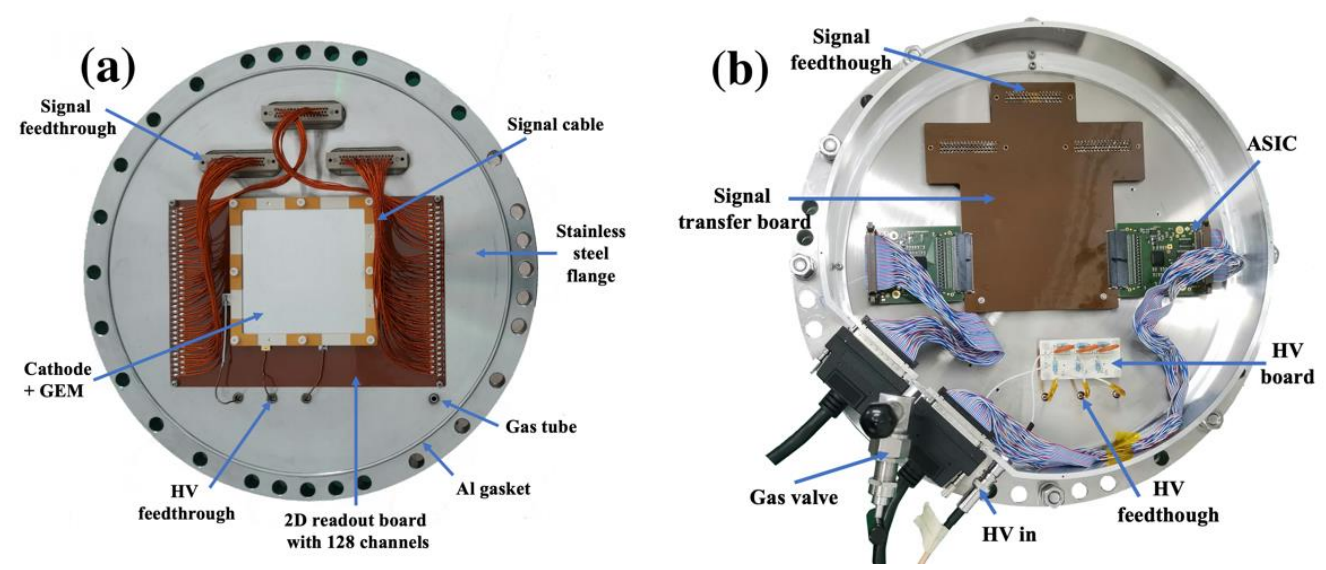

Fig. 2 (a) Photograph of gas volume. (b) Photograph of electronics chamber.

A special system was used for the detector outgassing: the detector was heated to $80^{\circ} \mathrm{C}$ to remove residual gas in the materials with heating tapes wrapped in $\mathrm{Al}$ foils for the heat uniformly. Under these conditions, the gas volume was vacuumed by the mechanical and molecular pumps for 5 days. During this period, the mixture gas of $\mathrm{Ar}$ and $\mathrm{CO}_{2}$ (ratio 9:1, purity >99.999\%) was used to flush the gas volume for serval times. There were not hydrogen or fluoride components which were 
prone to ageing [22] in the mixture gas. Finally, the mixture gas was flushed into the gas volume until the pressure reached $1.0 \mathrm{~atm}$.

\section{Experimental results}

The performances of the sealed ceramic GEM-based neutron detector, such as the plateau curve, the neutron beam profile, the neutron wavelength spectrum, the spatial resolution, the $2 \mathrm{D}$ imaging ability, the neutron detection efficiency and the counting rate stability, were studied using Time Of Flight (TOF) approach at BL20 of CSNS. The moderator was decoupled poisoned liquid hydrogen and the diameter of neutron beam exit was $20 \mathrm{~mm}$ in the BL20. A commercial neutron beam monitor, ORDELA 4562N (monitor), was placed at beam exit and measured simultaneously with the detector to eliminate neutron beam fluctuation in the experiments.

\subsection{Plateau curve}

The plateau curve was the counting rate curve with different working high voltage (HV). The counting rates were measured at $\mathrm{HV}$ from $-1100 \mathrm{~V}$ to $-1800 \mathrm{~V}$ to find the appropriate $\mathrm{HV}$ of the detector. Meanwhile, the counting rate of the monitor was also measured. The result was shown in Fig. 3, the normalized count was defined as the ratio of the counting rate of the detector to that of the monitor. The plateau slope of detector was $0.5 \% / 100 \mathrm{~V}$ with the plateau ranging from $-1550 \mathrm{~V}$ to $-1800 \mathrm{~V}$. The fluctuation within the plateau range was caused by the low counts of the monitor. The detector $\mathrm{HV}$ was set at $-1600 \mathrm{~V}$ to improve the stability of the detector in the subsequent experiments.

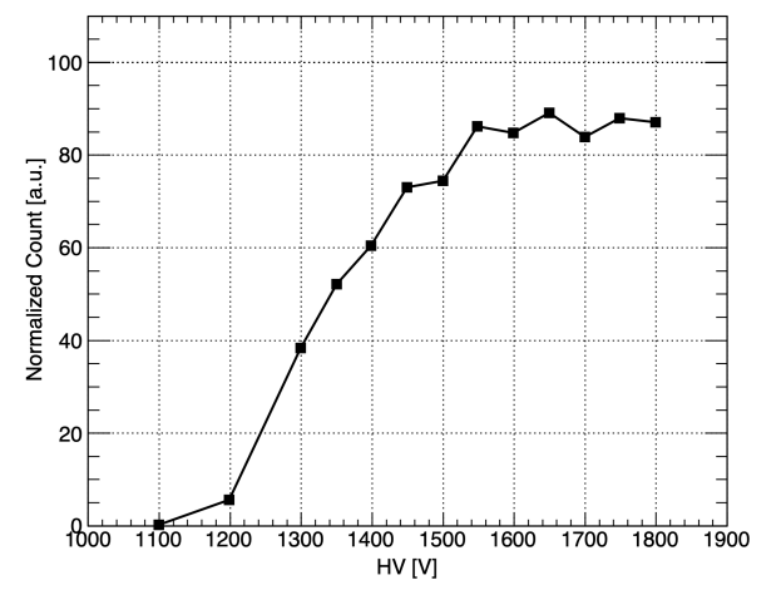

Fig. 3 Plateau curve of the detector. The plateau ranges from $-1550 \mathrm{~V}$ to $-1800 \mathrm{~V}$ and the plateau slope is $0.5 \% / 100 \mathrm{~V}$. Normalized count is defined as the ratio of the counting rate of the detector to that of the monitor.

\subsection{Neutron beam profile and neutron wavelength spectrum}

The neutron beam profile and the neutron wavelength spectrum were measured using the TOF method. Limited by the size of the detector, it could not be placed close to the beam exit and placed $1.4 \mathrm{~m}$ away from the beam exit to measure the direct beam. Due to the diffusion of neutron beam, the measured diameter of beam profile was about $36 \mathrm{~mm}$ which was larger than that of the beam exit (shown in Fig. 4(a)). To evaluate the neutron wavelength spectrum, the wavelength spectrum that the monitor measured was used as a comparison. Both of the spectrums from $0.5 \AA$ to $10 \AA$ were calibrated with their detection efficiency calculated by Geant4 [23] and then normalized to the 
maximum counts (shown in Fig. 4(b)). The spectrum shapes measured by the monitor and the detector were consistent with each other, and the result validated the accuracy of the detector measurement. For the neutrons with wavelengths from $2 \AA$ to $3 \AA$, the counts of detector were slightly lower than that of monitor. The $\mathrm{Al}$ incident window thickness of the monitor was $1 \mathrm{~mm}$ and that of the detector was $6 \mathrm{~mm}$, and there are four Bragg edges from $2 \AA$ to $3 \AA$ for the $\mathrm{Al}$ element, leading to the stronger Bragg diffraction intensity and lower counts of the detector.
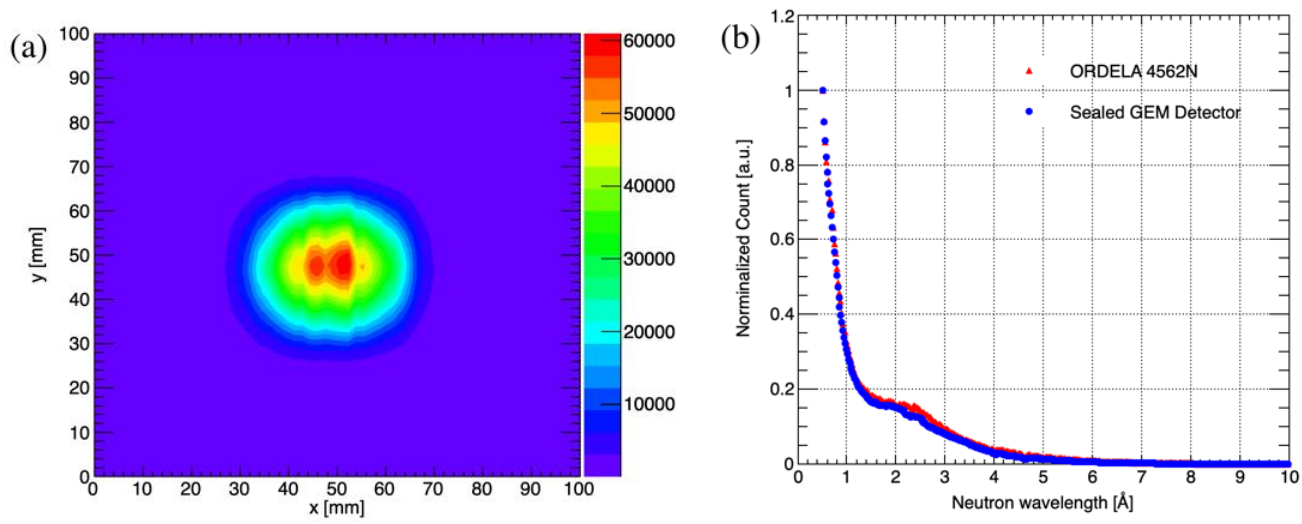

Fig. 4 (a) Imaging of neutron beam profile measured away from the beam exit $1.4 \mathrm{~m}$ at BL20 of CSNS. (b) Neutron wavelength spectrum of the detector compared with that of the monitor, both spectrums were calibrated with detection efficiency and normalized to the maximum counts.

\subsection{Spatial resolution and 2D imaging}

Spatial resolution was an important parameter for 2D position-sensitive neutron detector. The spatial resolution could be measured by the Line Spread Function (LSF) method with a sufficiently narrow slit, and it was defined as the Full-Width-Half-Maximum (FWHM) of the peak in the projection on $\mathrm{X}$-axis or Y-axis. A $2 \mathrm{~mm}$-thick Cadmium (Cd) mask, with three slits of $0.5 \mathrm{~mm}$ wide and $20 \mathrm{~mm}$ pitch, was employed. The Cd mask was mounted in the font of the incident window of detector, and the spatial resolution of $\mathrm{x}$ and $\mathrm{y}$ directions were measured by rotating the Cd mask 90 degrees. In order to obtain the similar counts for the three slits, the detector was positioned at the back end of the beam line, about $4.96 \mathrm{~m}$ away from the beam exit. The detector responses were shown in Fig. 5, and the FWHM was obtained by Gaussian fitting. The distances between all peaks were about $20 \mathrm{~mm}$, which was consistent with that of the Cd mask. The spatial resolution in $\mathrm{x}$ direction was below $2.77 \pm 0.01$ (stat) $\mathrm{mm}$ (FWHM) and that in y direction was below $2.55 \pm 0.01$ (stat) $\mathrm{mm}$ (FWHM). The detector had good spatial resolution, and the spatial resolution could be further improved by reducing the pitch of strips in 2D readout board. 

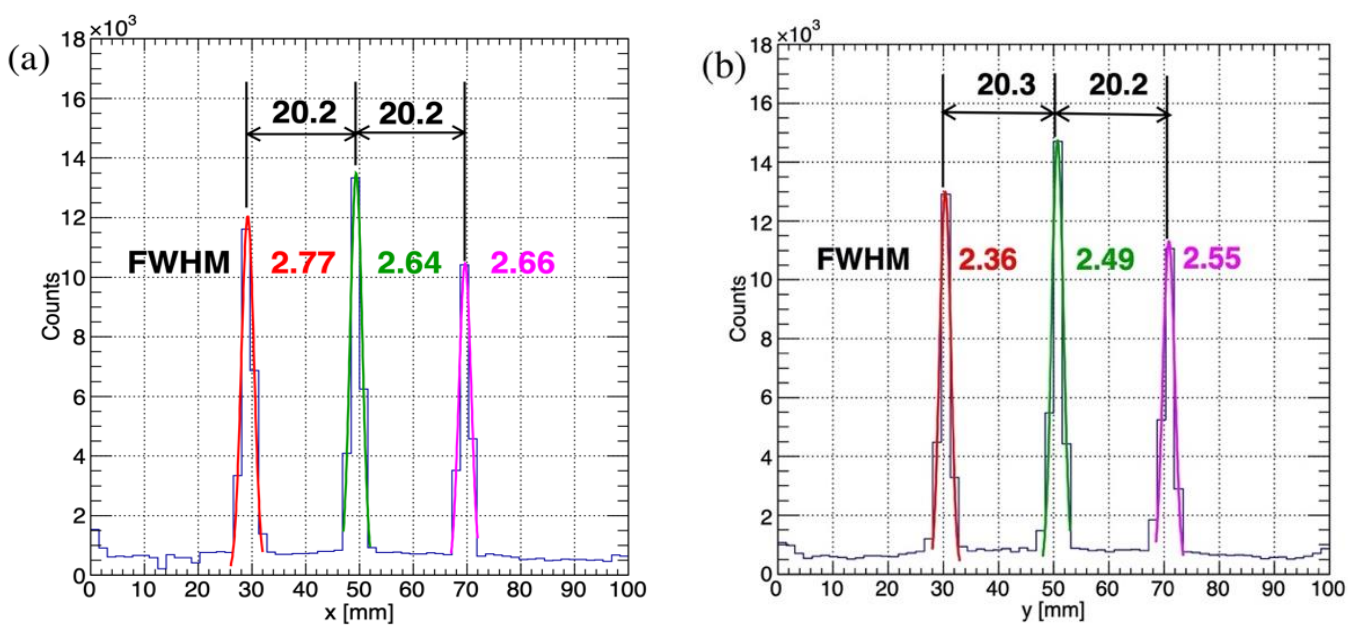

Fig. 5 Measurement of spatial resolution in X (a) and Y (b) directions. The Cd mask with three slits was employed, the slit width was $0.5 \mathrm{~mm}$ and the pitch was $20 \mathrm{~mm}$.

2D imaging was measured by a $2 \mathrm{~mm}$-thick Cd mask with a "CSNS" pattern (shown in Fig. 6(a)), and the imaging result was shown in Fig. 6(b). The four letters could be clearly distinguished which were consistent with the pattern and the background signal was low. In the imaging, the counts were high in the middle and low on the left and right sides, which was caused by the uneven neutron flux in the beam profile. The result indicated that the detector had a good capability of $2 \mathrm{D}$ imaging.
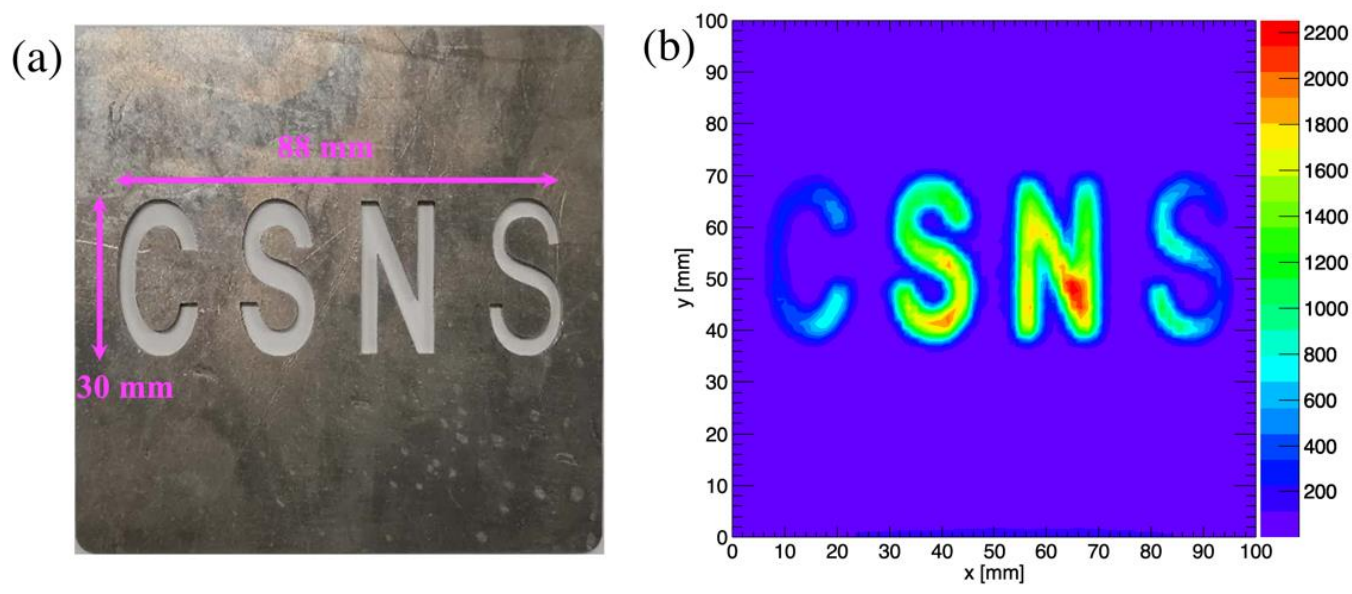

Fig. 6 (a) Cd mask with "CSNS" pattern, (b) the result of 2D imaging.

\subsection{Neutron detection efficiency}

The enriched $\mathrm{B}_{4} \mathrm{C}$ with 1.0 um thickness was employed as neutron converter. The neutron detection efficiency of the detector was evaluated by comparing with the standard ${ }^{3} \mathrm{He}$ counting tube (20 atm, 1 inch in diameter). And it was the ratio of the normalized count of the detector to that of the ${ }^{3} \mathrm{He}$ tube, the normalized count was defined as the ratio of the counts of detector or ${ }^{3} \mathrm{He}$ tube to that of monitor. After being deflected by MICA monochromator, the neutron beam was collimated by a $5 \mathrm{~mm}$ thick boron aluminum alloy plate with a small hole ( $2 \mathrm{~mm}$ in diameter). The hole was aligned with the center of the ${ }^{3} \mathrm{He}$ tube or the sensitive area of the detector. The neutron detection efficiency for $1.56 \AA$ was measured to $2.3 \pm 0.1$ (stat)\%. As a comparison, the relationship between 
the detection efficiency and the neutron wavelength was obtained by Geant4 (shown in Fig. 7). For the neutron with a wavelength of $1.56 \AA$, the simulation result of detection efficiency was $2.5 \%$. The deviation between the results of experiment and simulation might be due to the thickness of $\mathrm{B}_{4} \mathrm{C}$ and incident window.

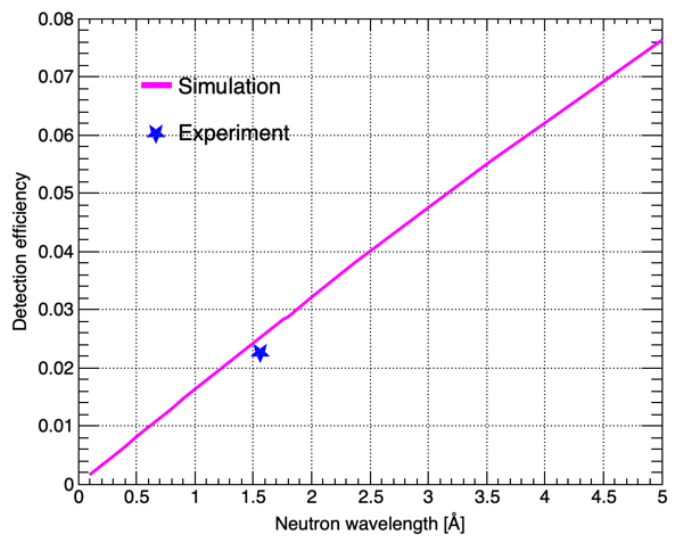

Fig. 7 Comparison of neutron detection efficiency between simulation and experiment. Neutron detection efficiency varies with neutron wavelength (calculated by Geant4, magenta curve). The experiment measurement (blue star).

\subsection{Counting rate stability}

The long-term stability is crucial for the sealed ceramic GEM-based neutron detector. In order to evaluate its counting rate stability, the detector was measured continuously for 17 hours and the data from the detector, the monitor and the proton targeting was collected every 20 minutes. The results were shown in Fig. 8. The counting rate of detector were normalized by the counting rate of monitor (blue triangle) and protons (red dot), and their instability fluctuations which were defined by RSD were $0.64 \%$ and $0.73 \%$, respectively. Both results showed that the detector had a good long-term counting rate stability.

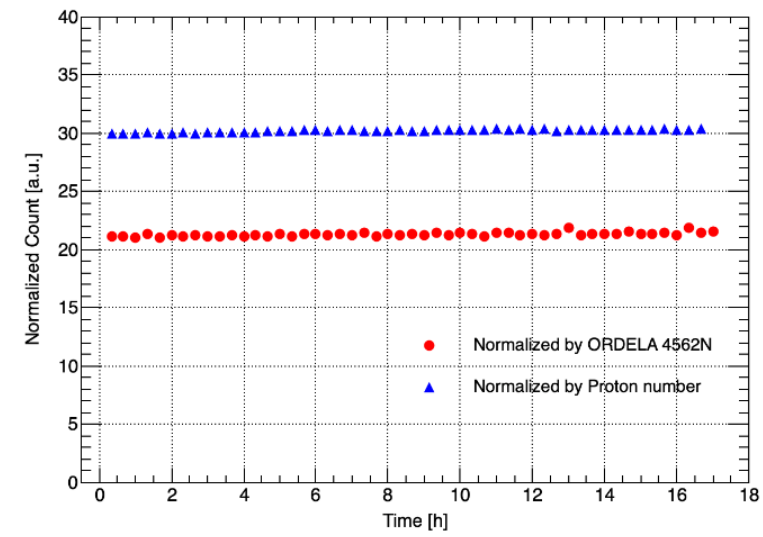

Fig. 8 Counting rate stability for 17 hours. The red dot represents the counts of the detector normalized by that of monitor, and the blue triangle represents the counts of the detector normalized by that of protons.

\section{Conclusion and outlook}


A sealed ceramic GEM-based neutron detector with a $100 \mathrm{~mm} \times 100 \mathrm{~mm}$ sensitive area was developed and measured at CSNS. The capability of 2D imaging and wavelength resolved was demonstrated. The plateau of detector ranged from $-1550 \mathrm{~V}$ to $-1800 \mathrm{~V}$ with $0.5 \% / 100 \mathrm{~V}$ slope, the spatial resolution was below $2.77 \pm 0.01 \mathrm{~mm}$ (FWHM), the detection efficiency for neutrons with a wavelength of $1.56 \AA$ was $2.3 \pm 0.1 \%$, the counts instability fluctuation was about $0.7 \%$ (RSD) in 17 hours of continuous measurement. The results show that the detector had good performances in sealed mode, and it could be used for neutron beam monitor, very small angle neutron scattering, neutron reflection and so on. In the future, a sealed cascade ceramic GEM-based neutron detector with high detection efficiency will be developed and used in the new instruments of CSNS.

\section{Acknowledgments}

This work was supported by the National Key R\&D Program of China [Grant No. 2017YFA0403702], the National Natural Science Foundation of China [Grant No. 11635012, 11775243, U1832119], Youth Innovation Promotion Association CAS [Grant No. Y8291480K2] and Guangdong Basic and Applied Basic Research Foundation [2019A1515110217].

\section{References}

[1] Chen, Hesheng, et al., Target station status of China Spallation Neutron Source, Neutron News 29 (2) (2018) 2-6.

[2] Gabriel, Tony A, STATUS REPORT ON THE SPALLATION NEUTRON SOURCE, 1998.

[3] Oyama, Yukio, J-PARC and new era of science, Nucl. Instrum. Methods Phy. Res. A 562 (2) (2006) 548-552.

[4] Price, D. L, Pulsed neutron sources for condensed-matter research, Argonne National Lab 1980.

[5] Åberg, M., et al, ESS technical design report, European Spallation Source 2013.

[6] Hendricks, Robert W., Space charge effects in proportional counters, Rev. Sci. Instrum. 40 (9) (1969) 1216-1223.

[7] Shea, Dana A., Daniel L. Morgan, The helium-3 shortage: Supply, demand, and options for congress, 2010.

[8] Sauli, Fabio, GEM: A new concept for electron amplification in gas detectors, Nucl. Instrum. Methods Phy. Res. A 386 (2-3) (1997) 531-534.

[9] Klein, Martin, Christian J. Schmidt, CASCADE, neutron detectors for highest count rates in combination with ASIC/FPGA based readout electronics, Nucl. Instrum. Methods Phy. Res. A 628 (1) (2011) 9-18.

[10] Shoji, M., et al., Development of GEM-based detector for thermal neutron, J. Instrum. 7 (05) (2012) C05003.

[11] Croci, G., et al., GEM-based thermal neutron beam monitors for spallation sources, Nucl. Instrum. Methods Phy. Res. A 732 (2013) 217-220.

[12] Croci, Gabriele, et al., Characterization of a thermal neutron beam monitor based on gas electron multiplier technology, Progress of Theoretical and Experimental Physics 2014 (8) (2014) $083 \mathrm{H} 01$.

[13] Cippo, E. Perelli, et al., A GEM-based thermal neutron detector for high counting rate applications, J. Instrum. 10 (10) (2015) P10003. 
[14] Köhli, M., et al., Efficiency and spatial resolution of the CASCADE thermal neutron detector, Nucl. Instrum. Methods Phy. Res. A 828 (2016) 242-249.

[15] Zhou, Jianrong, et al., A ceramic GEM-based neutron beam monitor for China Spallation Neutron Source. Nucl. Instrum. Methods Phy. Res. A 962 (2020) 163593.

[16] Zhou, Jianrong, et al., Highly efficient GEM-based neutron detector for China Spallation Neutron Source, Nucl. Instrum. Methods Phy. Res. A 953 (2020) 163051.

[17] Breskin, Amos, et al., A concise review on THGEM detectors, Nucl. Instrum. Methods Phy. Res. A 598 (1) (2009) 107-111.

[18] Zhou, Jianrong, et al., A novel ceramic GEM used for neutron detection, Nucl. Eng. Technol 52 (6) (2020) 1277-1281.

[19] Tamagawa, T., et al., Development of gas electron multiplier foils with a laser etching technique, 560 (2) (2006) 418-424.

[20] Takeuchi, Yoko, et al., Development and properties of $100 \mathrm{~mm}$-square size LTCCGEM, Journal of Physics: Conference Series, 1498 (1) 2020.

[21] Becker, J., et al., A vertex trigger based on cylindrical multiwire proportional chambers, Nucl. Instrum. Methods Phy. Res. A 586 (2) (2008) 190-203.

[22] Niebuhr, Carsten, Aging effects in gas detectors, Nucl. Instrum. Methods Phy. Res. A 566 (1) (2006) 118-122.

[23] Agostinelli, Sea, et al., GEANT4-a simulation toolkit, Nucl. Instrum. Methods Phy. Res. A 506 (3) (2003) 250-303. 